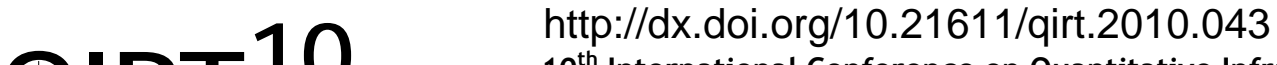 \\ $10^{\text {th }}$ International Conference on Quantitative InfraRed Thermography \\ July 27-30, 2010, Québec (Canada)
}

\section{Thermoelastic stress analysis of titanium components and simultaneous assessment of residual stress}

\author{
by U. Galietti, D. Palumbo* \\ * Politecnico di Bari, Dipartimento di Ingegneria Meccanica e Gestionale, Viale Japigia 182 Bari, Italy, \\ galietti@poliba.it, d.palumbo@poliba.it
}

\begin{abstract}
The thermoelastic effect describes a linear relationship between change in body temperature and state of stress in the presence of adiabatic conditions. This approach considers the material properties constant with temperature, which is not correct for all materials. Experimental results and a review of the theory, especially for the titanium and some aluminium alloys, have shown that the thermoelastic signal is also dependent of mean stress of the material. The use of titanium and aluminium in various high tech fields of application, like aerospace and biomechanics, is growing fast and so it is necessary to perform the thermoelastic stress analysis, that is a full field technique, with the best precision taking into account also second order effects. Hence, it is necessary to make a correction of the measurement in relation to the mean stress. The possibility to measure the mean stress allows also an evaluation of residual stresses on the surface of titanium and aluminium components.
\end{abstract}

\section{Introduction}

The Thermoelastic Stress Analysis (TSA), thanks to Focal Plane Array (FPA) differential cameras, is able to provide very quickly map of the sum of the principal stresses of components subjected to actual loading conditions. The thermoelastic theory, and subsequent experimental results [1], [2], [3], [4], [5], [6], [7], [8], have shown the possibility for some materials, to assess residual stresses. The thermographic signal, in fact, is a function of the mean stress and mechanical properties of the material [1], [2], [3], [5]. The thermographic signal depends on the variation of longitudinal modulus of elasticity with temperature [2], [3]. Whilst this effect has been experimentally shown in literature, the correction of the consequent error in application of TSA on titanium components has been proposed in this work together with a procedure able to assess in a quantitative way residual stresses.

The use of titanium and aluminium in various application fields, from aerospace to the automotive sector, is pushing research towards rapid and full field stress analysis techniques, as the thermoelastic technique, able to validate numerical models. If TSA will proof to be a mean to assess the surface residual stresses with an acceptable error, we would have one very powerful tool for the analysis, monitoring and control of components.

For many reasons, in the analysis of real components, it is often impossible to perform the tests with mean loads equal to zero. For example in the case of thin structures or complex structures, where there are buckling or clearance problems in the loading system. Therefore it is necessary for real structures a procedure which can obtain either the values of the thermoelastic parameters of the material performing a correction of the thermoelastic data and an assessment of residual stresses using variable loads, but with the same sign.

This work presents an innovative procedure for measuring the stress state of titanium components that takes into account the effects due to the mean stress, it will be shown that neglecting those effects could lead to errors larger than $20 \%$ according to the area used for experimental calibration of thermoelastic signal. Problems can raise in the industrial use of TSA for titanium and aluminium complex components. The presence of strong variation of sum of the principal stresses determine the difficulty to calibrate properly the thermoelastic signal.

The possibility to measure the mean stress allows the assessment of residual stresses on the surface of titanium and aluminium components with the thermoelastic technique [4]. A procedure for the evaluation of residual stress was developed. This procedure relies on the analysis of the stressed specimen in comparison with an annealed reference specimen. It could be the same component before and after annealing.

\section{Theory}

Thermoelastic stress analysis refers to the estimation of the state of stress in a structure by the measurement of the thermal response resulting from the application of a load within the elastic range of the material. This approach considers the material properties constant with temperature, which is not correct for all materials. The experimental works by Belgen (1968) and Machin et al [1], have show, for some materials such as titanium and some aluminium alloys, a dependency of the 
thermoelastic signal from the material mean stress. In the same period Wong et al [2], [3] presented a review of the thermoelastic theory by introducing higher-order terms that reveal the kind of link between temperature change and mean stress.

Expressing the equation of energy conservation for an isotropic material, in the case of small changes in temperature and adiabatic conditions and in the case of uniaxial stress with sinusoidal load gives [2], [3], [6], [7]:

$$
\rho_{0} C_{\varepsilon} \frac{\dot{T}}{T}=-\left[\alpha+\left(\frac{v}{E^{2}} \frac{\partial E}{\partial T}-\frac{1}{E} \frac{\partial v}{\partial T}\right) I_{1}\right] \dot{I}_{1}+\left[\frac{(1+v)}{E^{2}} \frac{\partial E}{\partial T}-\frac{1}{E} \frac{\partial v}{\partial T}\right] \sum_{i=1}^{3} \sigma_{i i} \dot{\sigma}_{i i}
$$

where $T$ is the thermodynamic temperature (Kelvin); $\sigma_{i i}$ are the principal stresses $\left(\mathrm{N} / \mathrm{m}^{2}\right) ; l_{1}$ is the sum of the principal stresses, $\left(\mathrm{N} / \mathrm{m}^{2}\right) ; \rho_{o}$ is the density $\left(\mathrm{kg} / \mathrm{m}^{3}\right) ; C \varepsilon$ is the specific heat under constant strain $\left(\mathrm{N} \mathrm{m} /{ }^{\circ} \mathrm{C} \mathrm{kg}\right) ; \alpha$ is the coefficient of thermal expansion $\left({ }^{\circ} \mathrm{C}^{-1}\right) ; E$ is the Young's modulus $\left(\mathrm{N} / \mathrm{m}^{2}\right)$; and $v$ is the Poisson's ratio. The dotted symbols represent derivatives with respect to time.

In the case of one dimensional stress with load sine we have:

$$
\sigma_{11}=I_{1}=\sigma_{m}+\sigma_{a m p} \sin \omega t \quad, \quad \sigma_{22}=\sigma_{33}=0
$$

where $\sigma_{m}$ and $\sigma_{a m p}$ are the mean and amplitude of the applied stress respectively and $\omega$ is the loading frequency. Substituting relations (2) in equation (1) yields:

$$
\rho_{0} C_{\varepsilon} \frac{\partial T}{T_{0}}=-\left(\alpha-\frac{1}{E^{2}} \frac{\partial E}{\partial T} \sigma_{m}\right) \Delta \sigma \sin \omega t-\frac{1}{4 E^{2}} \frac{\partial E}{\partial T}(\Delta \sigma)^{2} \cos 2 \omega t
$$

The equation above shows that the thermal response of a solid body subjected to a purely sinusoidal load is the algebraic sum of a first part with pulsation $\omega$ equal to the pulse of load, which is a function of both amplitude loading and the mean load, and a second part, with pulsation $2 \omega$.

As is known, the frequency correlation of the thermoelastic signal allows to filter the signal components at different frequency from the frequency load. In this case the temperature variation and the amplitude load are connected by thermoelastic parameter $K$ :

$$
K=\left(\alpha-\frac{1}{E} \frac{\partial E}{\partial T} \sigma_{m}\right)\left(\rho C_{\varepsilon}\right)^{-1}
$$

A normalised measure of the dependence of $\mathrm{K}$ on $\sigma_{\mathrm{m}}$ is thus:

$$
\frac{1}{K_{0}} \frac{\partial K}{\partial \sigma_{m}}=\frac{-1}{\alpha E^{2}} \frac{\partial E}{\partial T}
$$

where $K_{0}=\alpha /\left(\rho_{0} C_{p}\right)$ is the conventional thermoelastic constant.

This relationship shows that the variation of the thermoelastic parameter with the mean stress is due to the variation of Young's modulus with temperature.

The relationship (5) quantify, for different materials, the effect that mean stress has on thermography signal. Experimentally in literature [6], [7] this quantity may be evaluated separately diagrammed the first component at pulsation $\omega$ as a function of amplitude stress, and the second component at $2 \omega$ as a function of the square of the amplitude stress, a zero mean stress. The ratio between the slopes of two straight lines obtained provides, less than a factor of 4 , the amount sought. Good agreement in the literature [6], [7] was found with the theoretical and experimental results for titanium (Ti-6Al4V) and aluminium (Al-2024).

Neglecting the variations of Young's modulus $E$ and Poisson's ratio $v$ with temperature and expressing the specific heat at constant strain with the relationship:

$$
C_{\varepsilon}=C_{p}-\frac{3 E \alpha^{2} T}{1-2 v}
$$

the relation between the change temperature of a solid and the variation of the trace of stress tensor for a plane stress state is obtained: 


$$
\Delta T=-K_{0} T\left(\Delta \sigma_{1}+\Delta \sigma_{2}\right)
$$

with $K_{0}$ thermoelastic constant of the material.

Dulieu-Barton et al [8] have show differences in thermoelastic output from steel and aluminium components subjected to prior plastic strain in comparison to identical not deformed components. In particular has been show that the thermoelastic signal variation may be due to a change in the material's coefficient of thermal expansion. The variation of thermoelastic constant of material can be used for assessment of residual stress in components.

An experimental evaluation of eq. (5) analyzing only the first component of pulsation $\omega$ and an innovative procedure to correct the thermoelastic data and to estimate the residual stresses on titanium, will be done in the following paragraphs.

\section{Experimentation}

\subsection{Thermoelastic characterisation of material}

All the test have been performed on a Schenck pc400m servohydraulic fatigue machine with 250 kN capacity. The thermoelastic data have been acquired via the differential IR camera DeltaTherm 1560 (DT) made by StressPhotonics (USA) with thermal sensitivity (NEdT) $<18 \mathrm{mK}$ and based on a InSb photonic detector with 320×256 pixel.

Two specimens were manufactured from titanium (TI-6Al-4V) and one specimen in aluminium alloy (Al-2024) with dimensions and geometry shown in Figure (1) were tested. The titanium specimens were subjected to a annealing thermal cycle by treatment in an oven at a temperature of $735^{\circ} \mathrm{C} \pm 15^{\circ} \mathrm{C}$ in atmosphere containing argon, for 2 hours. Before the tests, specimens were painted with matt black paint to make uniform the emissivity of the treated surfaces and avoid reflections due to heat sources placed near the specimens during the test.

On the titanium unnotched specimen, called as specimen 1 and aluminium unnotched specimen, called as specimen 3, the tests are conducted for different values of the mean load varying also the amplitude load from a minimum value of 2500 [N] to a maximum value of 10000 [N] (Table 1 and Table 2). To assess bending out of plane due to an imperfect alignment of clamps of the machine loading, we used a mirror to frame simultaneously with the camera, front and rear surfaces of the specimen. All tests were performed at a load frequency of $10[\mathrm{~Hz}]$ and three replications were made for each measurement.

As it can be seen in table 1 tests were also performed with a null mean load on specimen 1 . These data were not used in the proposed procedures but they are used as control data for verifying the results.

Table 1. Test performed on the titanium specimens. (Three replications were made for each measurement).

\begin{tabular}{|c|c|c|c|c|c|c|}
\hline & \multicolumn{4}{|c|}{ specimen 1} & \multicolumn{2}{|c|}{ specimen 2} \\
\hline Test & $\begin{array}{l}\mathrm{Fm} \\
{[\mathrm{N}]}\end{array}$ & $\begin{array}{c}\sigma m \\
{[\mathrm{MPa}]}\end{array}$ & $\begin{array}{l}\Delta \mathrm{F} \\
{[\mathrm{N}]}\end{array}$ & $\begin{array}{c}\Delta \boldsymbol{\sigma} \\
{[\mathrm{MPa}]}\end{array}$ & $\begin{array}{l}\text { Fm } \\
{[\mathrm{N}]}\end{array}$ & $\begin{array}{l}\Delta \mathrm{F} \\
{[\mathrm{N}]}\end{array}$ \\
\hline 1 & \multirow{4}{*}{15000} & \multirow{4}{*}{139} & 2500 & 22 & 5000 & \multirow{12}{*}{5000} \\
\hline 2 & & & 5000 & 44 & 7500 & \\
\hline 3 & & & 7500 & 66 & 10000 & \\
\hline 4 & & & 10000 & 88 & 12500 & \\
\hline 5 & \multirow{4}{*}{30000} & \multirow{4}{*}{263} & 2500 & 22 & 15000 & \\
\hline 6 & & & 5000 & 44 & 17500 & \\
\hline 7 & & & 7500 & 66 & 20000 & \\
\hline 8 & & & 10000 & 88 & 22500 & \\
\hline 9 & \multirow{4}{*}{45000} & \multirow{4}{*}{395} & 2500 & 22 & 25000 & \\
\hline 10 & & & 5000 & 44 & 27500 & \\
\hline 11 & & & 7500 & 66 & 30000 & \\
\hline 12 & & & 10000 & 88 & 32500 & \\
\hline 13 & \multirow{2}{*}{0} & \multirow{2}{*}{0} & 2500 & 22 & & \\
\hline 14 & & & 5000 & 44 & & \\
\hline
\end{tabular}

Table 2. Test performed on the aluminium specimen. (Three replications were made for each measurement).

\begin{tabular}{|c|c|c|c|c|}
\hline Test & $\begin{array}{l}\mathrm{Fm} \\
{[\mathrm{N}]}\end{array}$ & $\begin{array}{c}\sigma m \\
{[\mathrm{MPa}]}\end{array}$ & $\begin{array}{l}\Delta \mathbf{F} \\
{[N]}\end{array}$ & $\begin{array}{c}\Delta \sigma \\
{[\mathrm{MPa}]}\end{array}$ \\
\hline 1 & \multirow{4}{*}{10000} & \multirow{4}{*}{50} & 2500 & 13 \\
\hline 2 & & & 5000 & 25 \\
\hline 3 & & & 7500 & 38 \\
\hline 4 & & & 10000 & 50 \\
\hline 5 & \multirow{4}{*}{20000} & \multirow{4}{*}{101} & 2500 & 13 \\
\hline 6 & & & 5000 & 25 \\
\hline 7 & & & 7500 & 38 \\
\hline 8 & & & 10000 & 50 \\
\hline 9 & \multirow{4}{*}{30000} & \multirow{4}{*}{151} & 2500 & 13 \\
\hline 10 & & & 5000 & 25 \\
\hline 11 & & & 7500 & 38 \\
\hline 12 & & & 10000 & 50 \\
\hline
\end{tabular}




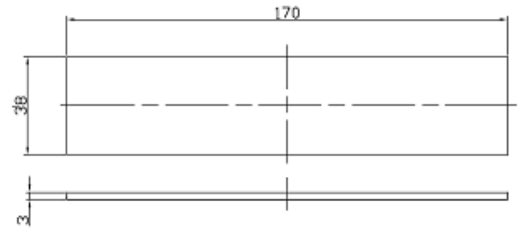

a)

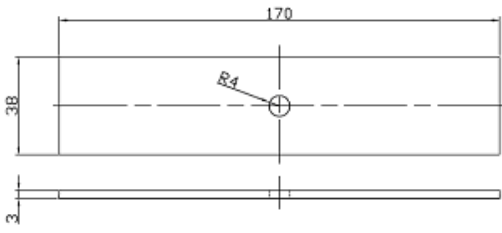

b)

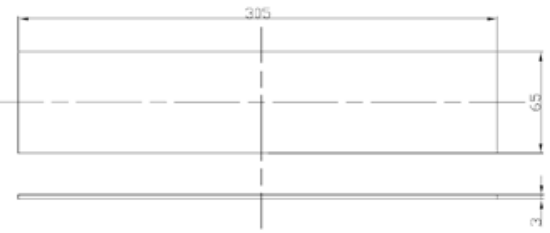

c)

Fig 1. Dimensions and geometry of specimens: a) unnotched titanium specimen (specimen 1); b) titanium notched specimen (specimen 2); c) aluminium unnotched specimen (specimen 3)

\subsection{Data correction and residual stress evaluation}

On the titanium notched specimen (specimen 2), the tests were conducted by varying the mean load from a minimum value of 5000 [N] to a maximum value of 32500 [N], (mean nominal stress equal respectively to 44 [MPa] and 285 [MPa]) keeping the amplitude constant load equal to $5000[\mathrm{~N}]$.

\section{Experimental data and results}

Thermoelastic stress analysis provides a signal $S$ proportional to the peak to peak variation in temperature during the variation peak to peak of the sum of stress $\Delta \sigma$. DeltaTherm provides the signal in the single frequency reference signal, filtering the second order signal frequency (Eq. 3). So defining:

$$
a=\frac{\alpha}{\rho C_{\varepsilon}}, \quad b=\frac{1}{E^{2}} \frac{\partial E}{\partial T}\left(\rho C_{\varepsilon}\right)^{-1}
$$

In this work it is proposed to analyse all data in the following form. From equation (3) we obtain:

$$
\Delta T / T_{0}=S_{T}=\left[a+b\left(\sigma_{m}+\sigma_{r}\right)\right] \Delta \sigma
$$

with $S_{T}$ equal to the thermoelastic signal calibrated in terms of $\Delta T$ and normalized to the absolute temperature of the specimen and $\sigma_{m}, \Delta \sigma$ and $\sigma_{r}$ respectively mean, amplitude and residual stress.

\subsection{Assessment of thermoelastic parameters}

The specimen 1 and specimen 3 are used for the evaluation of the thermoelastic signal when the mean load varies for a given value of constant load amplitude.

$\Delta T$ and $T$ in equation 9 are obtained as average of respectively the TSA and thermographic signal, calibrated in temperature, of the mean values measured in areas 1 and 2 (front and rear) of the specimen in order to avoid any influence of bending moment due to loading system, Figure 2.

Since the specimen is annealed $\sigma_{r}=0$, this yields:

$$
S_{T}=\left[a+b \sigma_{m}\right] \Delta \sigma
$$

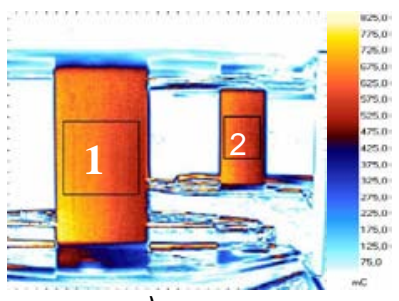

a)

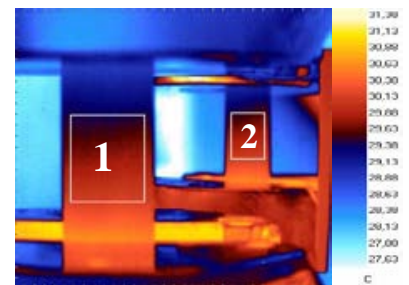

b)

Fig 2. Evaluation of the thermoelastic (a) (AC image) and thermographic signal (b) (DC image) 
In particular, a normalized signal/amplitude stress (Snorm $=\mathrm{S}_{T} / \Delta \sigma$ ) vs. mean stress curve is constructed for the evaluation of the constants that link the thermoelastic signal with the state of stress of the material. Figure 3 shows the values refer to tests performed on the specimen 1 and specimen 3.

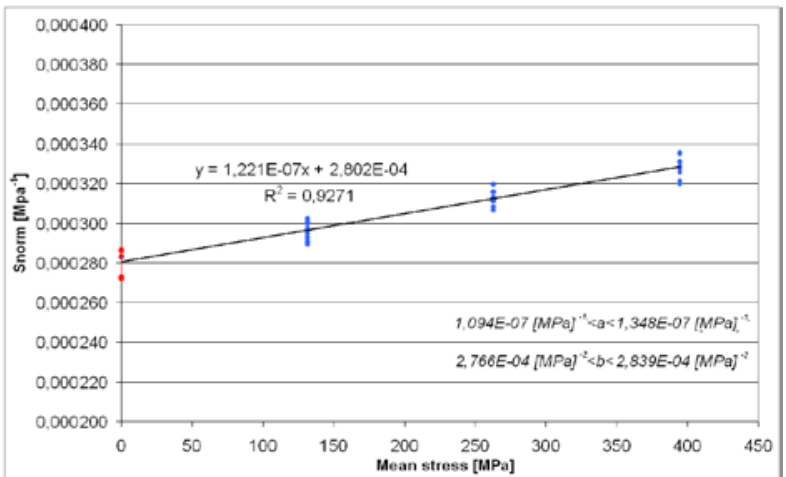

a)

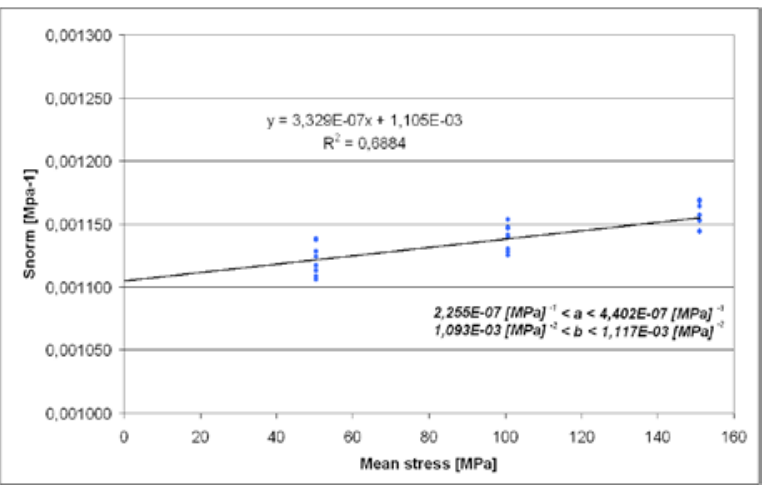

b)

Fig 3. Influence of mean stress on normalized thermoelastic signal: a) specimen 1 (titanium TI-6Al-4V) (the red data represents the zero mean stress tests); b) specimen 3 (aluminium Al-2024)

Figure 3 show the linear influence of mean stress on thermoelastic signal. Linear least squares fits to these data allows to derive the constants $a$ and $b$ in order to assess the relationship:

$$
\frac{b}{a}=\frac{1}{\alpha E^{2}} \frac{\partial E}{\partial T}
$$

The data in the literature [5] allow the calculation of the theoretical ratio $b / a$ for the titanium alloy Ti-6Al-4V and aluminium alloy Al-2024. For TI-6Al-4V putting $\alpha=9.0 \mathrm{E}-06\left[{ }^{\circ} \mathrm{C}^{-1}\right], \mathrm{E}=1.11 \mathrm{E}+05[\mathrm{MPa}]$ and $|\delta \mathrm{E} / \delta \mathrm{T}|=48.0\left[\mathrm{MPa} /{ }^{\circ} \mathrm{C}^{-1}\right]$ gives a ratio $\mathrm{b} / \mathrm{a}=4.33 \mathrm{E}-04\left[\mathrm{MPa}^{-1}\right]$ compared to an experimental value of $4.36 \mathrm{E}-04\left[\mathrm{MPa}^{-1}\right]$ with an error of about $1.4 \%$. For aluminium alloy 2024 putting $\alpha=2.3 \mathrm{E}-05\left[{ }^{\circ} \mathrm{C}^{-1}\right], \mathrm{E}=7.2 \mathrm{E}+04[\mathrm{MPa}]$ and $|\delta \mathrm{E} / \delta \mathrm{T}|=36.0\left[\mathrm{MPa} /{ }^{\circ} \mathrm{C}^{-1}\right]$ gives a ratio b $/ \mathrm{a}$ $=3.02 \mathrm{E}-04\left[\mathrm{MPa}^{-1}\right]$ compared to an experimental value of 3.01E-04 [ $\left.\mathrm{MPa}^{-1}\right]$. Excellent agreement between theory and experiment is clearly evident [2], [3], [4], [5].

The intercept a represents the normalized signal value when the mean stress is zero. Its value for specimen1, obtained from the analyses already shown (fig. 3) was compared with $\mathrm{a}_{0}=2,790 \mathrm{E}-04\left[\mathrm{MPa}^{-1}\right]$ that is the average of the measurements obtained by performing the tests at zero mean stress. The difference between these values is less than $0.6 \%$. This means that the zero mean stress tests could be omitted to avoid possible buckling problems.

\subsection{Correction of thermoelastic data on titanium alloy}

The instruments used in the TSA are capable of measuring the temperature variation $\Delta T$ rather than the absolute temperature, and provide an output signal $S$ proportional to the variation of flow of photons emitted from the body and linked to the sum of principal stress by the relationship:

$$
S A=\Delta \sigma
$$

where $A$ is the calibration factor depending on the material, type of instrument used and environmental conditions of the test. The calibration of thermoelastic signal is needed to determine $A$ and evaluate $\Delta \sigma$ [9]. Classical experimental calibration procedure was performed on specimen 1 by evaluating the signal $S$ in an area where $\Delta \sigma$ is known. So it is possible to determine $A$ as $A=S / \Delta \sigma$. This value is obtained under the hypothesis that mean stress effect is negligible bringing to significant errors for titanium and aluminium alloys.

In this paper we propose a calculation procedure that can quantitatively assess the values of amplitude stress $(\Delta \sigma)$ taking into account the values of mean stress $\left(\sigma_{m}\right)$. In this regard, we consider a specimen without residual stresses subjected to a mean load $F m$ and amplitude load $\Delta F$ notes. Moreover, the constants a and b were previously obtained, so in linear elasticity condition, we can write:

$$
\sigma_{m}=k F m \text { e } \Delta \sigma=k \Delta F
$$


being $k$ a function of geometry of the component, so imposing:

$$
F m / \Delta F=\sigma_{m} / \Delta \sigma=\gamma
$$

and substituting equation (10)

$$
\text { b } \gamma \Delta \sigma^{2}+a \Delta \sigma-S_{T}=0
$$

that allows to obtain both the map of $\Delta \sigma$ that the map of $\sigma_{m}$ from eq. (14).

The proposed algorithm is described in Figure 4. For a given loading condition is acquired thermoelastic image and thermographic image of the sample. The relationship between the two images provides the normalized signal $S_{T}$. Equation (15), known constants a e $b$, allows to find the correct values of $\Delta \sigma$.

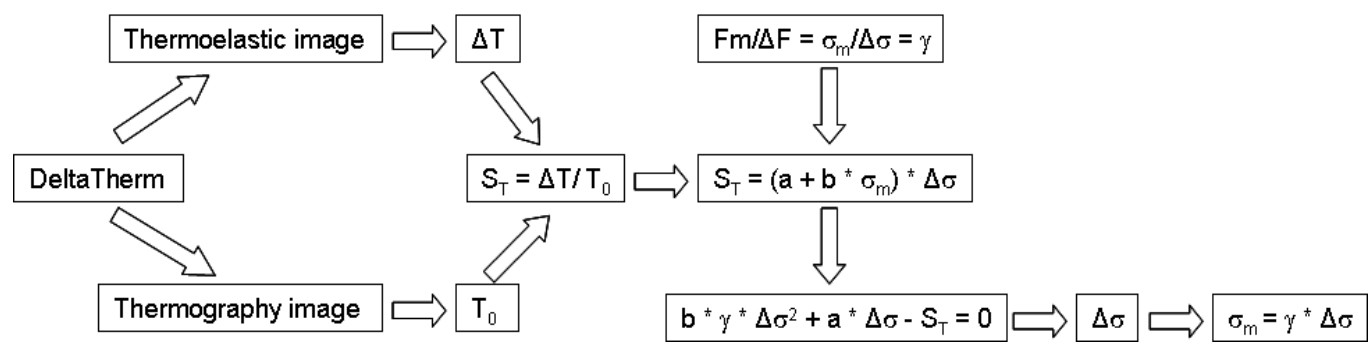

Fig 4. Algorithm proposed for the correction thermoelastic signal procedure

We show the results obtained using the algorithm proposed on the specimen 2 for loading conditions shown in Table 1. A comparison between the values of $\Delta \sigma$ obtained with the classical calibration procedure and with the new proposed algorithm was made.

The assessment of $A$ was performed on the specimen 1 evaluating average thermoelastic signal $S$ with a procedure similar to that of the previous paragraph. In particular, with zero mean load and amplitude load $\Delta \mathrm{F}=5000$ [N] by applying eq. (12) the value $A=5,75 \mathrm{E}-03[\mathrm{MPa} /$ unit signal] was obtained.

The effect of single stress state calibration can be assessed considering either a uniform stress profile (line 1 in figure. 5) and the high stress gradient profile (line 2 in figure. 5). The comparison between the two calibration procedures has been made along the profiles. So in the first case we compare the values of $\Delta \sigma_{\max }$ while in the latter case we compare the values of mean amplitude stress $\left(\Delta \sigma_{\mathrm{m}}\right)$. The errors for different values of the mean load and then for different values of the mean nominal stress at constant load amplitude $(\Delta \mathrm{F}=5000[\mathrm{~N}])$, (the asterisk indicates the results obtained from the algorithm proposed), are show in Table (3).

\begin{tabular}{|c|c|c|c|c|c|c|c|c|}
\hline TEST & $\begin{array}{l}\mathrm{Fm} \\
{[\mathrm{N}]}\end{array}$ & \begin{tabular}{|l}
$\sigma \mathrm{om}$ \\
nom. \\
(line 1) \\
[MPa] \\
\end{tabular} & $\begin{array}{l}\Delta \sigma_{\mathrm{m}} \\
\text { TSA } \\
\text { (line 1) } \\
{[\mathrm{MPa}]}\end{array}$ & $\begin{array}{l}\Delta \sigma_{\mathrm{m}}{ }^{*} \\
\text { corrected } \\
\text { (line 1) } \\
{[\mathrm{MPa}]}\end{array}$ & $\begin{array}{l}\text { Error } \\
\text { line1 } \\
\%\end{array}$ & $\begin{array}{l}\Delta \sigma_{\max } \\
\text { TSA } \\
\text { (line 2) } \\
\text { [MPa] }\end{array}$ & $\begin{array}{l}\Delta \sigma_{\max }{ }^{*} \\
\text { corrected } \\
\text { (line 2) } \\
{[\mathrm{MPa}]}\end{array}$ & \begin{tabular}{|l} 
Error \\
line2 \\
$\%$
\end{tabular} \\
\hline 1 & 5000 & 43,9 & 46,7 & 46,4 & 0,7 & 112,8 & 114,1 & $-1,2$ \\
\hline 2 & 7500 & 65,8 & 46,4 & 47,6 & $-2,4$ & 117,0 & 115,9 & 0,9 \\
\hline 3 & 10000 & 87,7 & 47,1 & 47,6 & $-1,2$ & 116,9 & 112,7 & 3,6 \\
\hline 4 & 2500 & 109,7 & 48,0 & 47,8 & 0,4 & 121,0 & 113,3 & 6,4 \\
\hline 5 & 5000 & 131,6 & 48,7 & 48,0 & 1,4 & 122,4 & 112,2 & 8,4 \\
\hline 6 & 7500 & 153,5 & 49,2 & 47,9 & 2,6 & 124,5 & 111,6 & 10,4 \\
\hline 7 & 10000 & 175,4 & 49,1 & 47,2 & 3,8 & 125,8 & 110,2 & 12,4 \\
\hline 8 & 2500 & 197,4 & 50,7 & 48,0 & 5,2 & 132,6 & 113,0 & 14,8 \\
\hline 9 & 5000 & 219,3 & 51,4 & 48,1 & 6,4 & 135,4 & 112,8 & 16,7 \\
\hline 10 & 7500 & 241,2 & 50,5 & 46,8 & 7,3 & 138,6 & 112,9 & 18,5 \\
\hline 11 & 10000 & 263,2 & 52,0 & 47,5 & 8,7 & 148,3 & 116,9 & 21,2 \\
\hline 12 & 2500 & 285,1 & 52,9 & 47,7 & 9,9 & 153,0 & 117,7 & $\underline{23,1}$ \\
\hline
\end{tabular}

Table 3. Comparison between the two calibration procedures along the considered profiles. 


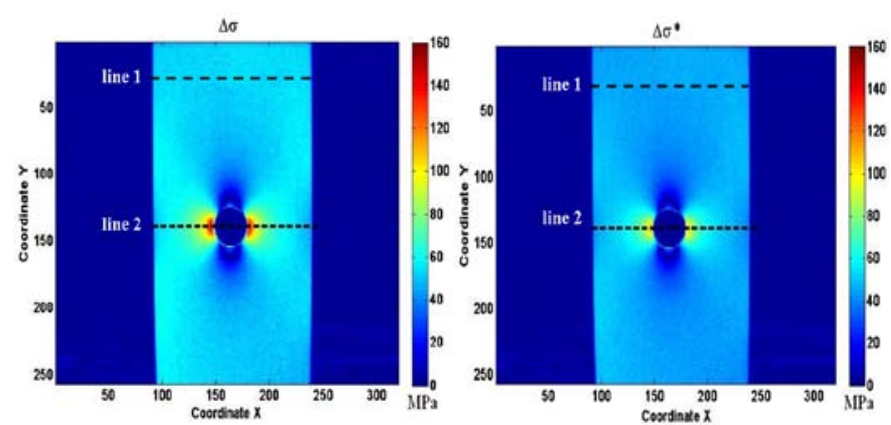

a)

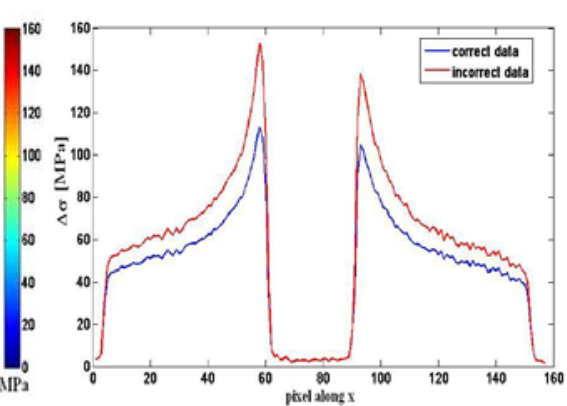

b)

Fig 5. a) map of amplitude stress obtained from incorrect $(\Delta \sigma)$ and correct data $\left(\Delta \sigma^{*}\right)$ b) Comparison between algorithm results ( $\triangle \sigma^{*}[\mathrm{MPa}]$, correct data) and classical calibration ( $\sigma$ [MPa], incorrect data)

The errors were assessed through relations:

$$
\text { Error line } 1 \%=100 *\left(\Delta \sigma_{m}-\Delta \sigma_{m}^{*}\right) / \Delta \sigma_{m} \quad \text { and } \quad \text { Error line } 2 \%=100 *\left(\Delta \sigma_{\max }-\Delta \sigma_{\max } *\right) / \Delta \sigma_{\max }
$$

As is shown in Table 3, the error is evident for values of the mean load exceeding 15000 [N], reaching maximum values around $10 \%$ and $23 \%$ respectively in areas with $\Delta \sigma$ constant and $\Delta \sigma_{\max }$.

\subsection{Assessment of residual stress on titanium}

A procedure for the evaluation of residual stress was also developed. This procedure relies on the analysis of the stressed specimen in comparison with an annealed reference specimen. Such procedure will allow two main analyses: monitoring of components for plastic deformation due to overstress of incipient fatigue failure and for assessing different manufacturing process in terms of residual stresses produced.

Assuming linear elasticity condition, known loads $\mathrm{Fm}$ and $\Delta \mathrm{F}$ and the constants $\mathrm{a}$ and $\mathrm{b}$, we can to trace back to the values of k pixel by pixel, known $\Delta \sigma$, eq. (13), (14) e (15).

Rewriting equation (9) as a function of $\mathrm{k}$, assuming linear elasticity and a general known external loads applied Fm and $\Delta \mathrm{F}$, we can write for the same specimen in the presence of residual stresses:

$$
S_{T}=\left(a+b \sigma_{r}\right) k \Delta F+b k^{2} F m \Delta F
$$

if $\mathrm{k}$ is known, it is immediate to obtain $\sigma_{r}$ as:

$$
\sigma_{r}=\left(\frac{S_{T}}{k \Delta F}-a\right) \frac{1}{b}-k F m
$$

In order to validate the residual stress analysis procedure a fake residual stress distribution similar to mean stress distribution was simulated by adding an extra known static load to the test mean load.

It means that Thermoelastic Stress Analysis was performed with the following loading conditions: Fm $=20000$ [N], $\Delta \mathrm{F}=5000[\mathrm{~N}]$ but the procedure proposed in par. 4.2 was instead used in order to determine the mean stress distribution imposing a different loading condition i.e. $\mathrm{Fm}=16000[\mathrm{~N}]$ and $\Delta \mathrm{F}=5000[\mathrm{~N}]$. This allowed us to simulate a distribution of fake residual stress that is consequence of the distribution of mean stress due to a static load of 4000[N] (difference between real and simulated mean load).

The results show clearly that the map of residual stresses is qualitatively coincident to the map of the mean stresses obtained with the procedure and quantitatively coincident with the expected values of mean stresses for a mean load of 4000 [N] (figure. 6). It must be remarked that the map of stress obtained is of extraordinary quality since, working on the same image, we get a high signal to noise ratio. For real components lower quality date are expected.

\section{Conclusions}

The potential of the thermoelastic technique in the evaluation of residual stresses and a procedure, for evaluate the exact value of $\Delta \sigma$ through a correction of the thermoelastic signal, was presented in this work. First there were evaluated the 
thermoelastic parameters that link the thermoelastic signal, normalized to the absolute temperature, at the values of stress amplitude and mean stress. After it was examined, by comparison, the error that can be made by calibrating the thermoelastic signal neglecting the influence of mean stress. In particular, on titanium notched specimen in the areas of highest stress, errors more than $20 \%$ were obtained. This means that the proposed procedure for correction of the thermoelastic signal is necessary for the validation of numerical models.

The possibility to measure the mean stress allows us to evaluation also residual stresses on the surface of titanium and aluminium components with the thermoelastic technique. In particular the new procedure proposed allows to assess in quantitative way residual stress with prior knowledge of an annealed reference specimen.

A new algorithm for the separate assessment of the mean stress and residual stress and has been developed further experimental test are actually on course. The aim of future work is to develop a calculation procedure that can estimate the residual stresses without any knowledge of the annealed component conditions.

However, the proposed procedure for comparison may be a very effective method of monitoring and control of surface residual stress components in fact, performed the test to start of the service, we could monitor the progress of stress over time.

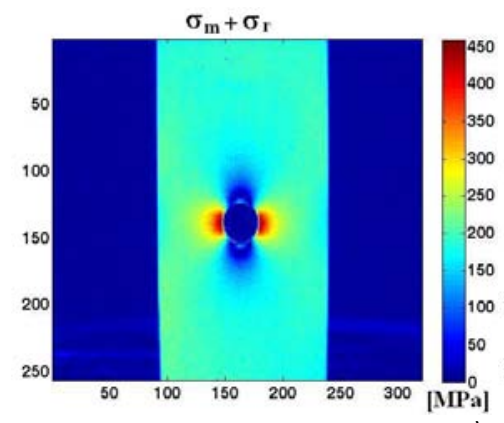

a)

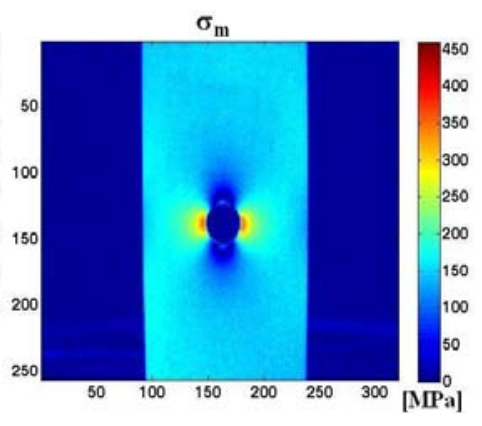

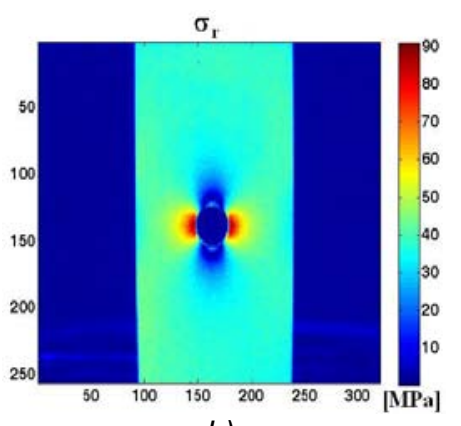

b)

Fig 6. a) map of mean stress on the specimen and identical specimen with and without residual stress; b) map of residual stress obtained with proposed procedure

\section{REFERENCES}

[1] Machin A.S., Sparrow J.G., Stimson M.G., "Mean stress dependence of the thermoelastic constant", Strain, 23, p. 27-30, 1987.

[2] Wong A.K., Jones R., Sparrow J.G., "Thermoelastic constant or thermoelastic parameter?", Journal of Physics and chemistry of solids, 48, p. 749-753, 1987.

[3] Wong A.K., Sparrow J.G., Dunn S.A., "On the revised theory of the thermoelastic effect", Journal of Physics and chemistry of solids, 49, p. 395-400, 1988.

[4] Wong A.K., Dunn S.A., Sparrow J.G., Nature, "Residual stress measurement by means of the thermoelastic effect", 332, p. 613-615, 1988.

[5] Dunn S.A., Lombardo D., Sparrow J.G., "The Mean Stress Effect in Metallic Alloys and Composites", SPIE, 1084, 129-142, 1989.

[6] Gyekenyesi A.L., Baaklini G.Y., "Thermoelastic Stress Analysis: The mean stress effect in metallic alloys", NASA/TM, 1999.

[7] Gyekenyesi A.L., Baaklini G.Y., "Quantifying Residual Stress by Means of Thermoelastic Stress Analysis", NASA/TM, 2001.

[8] Quinn S., Dulieu-Barton J.M. and Langlands J.M., "Progress in thermoelastic residual stress measurement", Strain, 40, p. 127-133, 2004.

[9] Dulieu-Smith S.M., "Alternative calibration techniques for quantitative thermoelastic stress analysis", Strain, 31, p. 9-16, 